\title{
Evaluation of OCPP and IEC 61850 for Smart Charging Electric Vehicles
}

\author{
Jens Schmutzler ${ }^{1}$, Claus Amtrup Andersen ${ }^{2}$, Christian Wietfeld ${ }^{1}$ \\ ${ }^{1}$ Dortmund University of Technology, Communication Networks Institute, Dortmund, Germany, \\ EMails: jens.schmutzler@tu-dortmund.de, christian.wietfeld@tu-dortmund.de \\ ${ }^{2}$ EURISCO Research \& Development, Odense, Denmark, EMails: caa@eurisco.dk
}

\begin{abstract}
Interoperability of charging infrastructures is a key success factor for E-Mobility. Standards like ISO/IEC 15118 and IEC 61851-1 are developed to ensure base level interoperability of front-end communication and signaling processes for smart charging between electric vehicles and charge spots. With the Open Charge Point Protocol (OCPP) a forum of European industry members also moves towards a common back-end protocol for charge spots intending to reduce and secure overall investment costs. However, in the current form OCPP lacks means for enabling grid services based on smart charging. In this paper the authors provide a review of today's state of the art in ISO/IEC standardization of the V2G Interface and furthermore detail how OCPP could leverage existing standardization efforts for grid automation from IEC 61850 in order to overcome its shortcomings.
\end{abstract}

Keywords: Smart Charging, OCPP, IEC 61850, ISO/IEC 15118, Vehicle-to-Grid Communication Interface, V2G

\section{Introduction}

With the introduction of EVs certain challenges arise for the power grid since it was deployed at times, when such additional loads were not yet considered. Assuming mass market adoption of EVs this inevitably comes along with high chances for concurrent charging processes and therefore overload of low voltage grid segments. In order to address these issues and ensure interoperability between charging infrastructures of various vendors, standardization bodies like ISO and IEC are busy defining corresponding standards for E-Mobility. As of today, core efforts were spent in front-end issues of E-Mobility, like plugs, in- \& outlets, electrical and safety requirements, as well as the front-end communication interface in order to ensure common access to EV charging infrastructures. Back-end and grid integration approaches were developed individually as part of research and pilot projects leading to proprietary solutions reflecting the situation on today's market. However, in terms of operational aspects as well as for securing the industry's investments in charging infrastructures it may be beneficial to standardize back-end integration aspects of charging infrastructures in the long term. According to the European Commission's climate and energy targets set in 2007 as well as following directives like 2009/28/EC [1] on the use of energy from renewable sources, EVs are considered to provide their share reducing $\mathrm{CO} 2$ emissions in the transport sector. Being shiftable loads as well as regularly available energy storages in the low-voltage grid, they are furthermore supposed to provide a certain benefit to grid stability in conjunction with increased penetration of renewable energy sources. In that sense the integration of EVs and charging infrastructures need to be extended beyond the current scope of purely operational aspects being covered by today's solutions. In order to integrate seamlessly in tomorrow's grid infrastructures a look into the IEC standardization roadmap [2] is necessary. They define IEC 61850 as core standard for grid automation and consider it as the core driver of Smart Grid development in the mid- and long-term.

Hence this work analyzes the alignment of the Open Charge Point Protocol (OCPP) - today's 
most commonly used and openly available backend communication protocol for charging infrastructures - and IEC 61850 in order to overcome today's lack of grid integration for charging infrastructures of EVs. In sections 3 and 4 both approaches for back-end integration are evaluated and differentiated from each other. In section 5 the authors summarize the lessons learned and provide an outlook on smart charging using OCPP and IEC 61850. But before going into such detail, some background on operational aspects of charging infrastructures and how plugged-in EVs can be characterized as grid assets is needed. Hence, the following section provides an overview on current state of the art considering the E-Mobility V2G Interface.

\section{Standardization Landscape of the E-Mobility V2G Interface}

Standardization for the V2G Interface in EMobility already has a long history which lately experienced major increase of interest and development. The global interest in reducing $\mathrm{CO} 2$ emissions in the transport sector and its resulting economical interests impacting two of the worlds largest industrial domains (utilities and automotive) led to extensive industrial support in research and development. The resulting standardization landscape of E-Mobility can be classified in the following four domains:

\section{Plugs (in- \& outlets)}

2. Charging Topologies

3. Communication

4. Security

5. Safety

The remainder of this section will walk through each domain highlighting corresponding ISO/IEC standardization work. An overview of the landscape is shown in Figure 1.

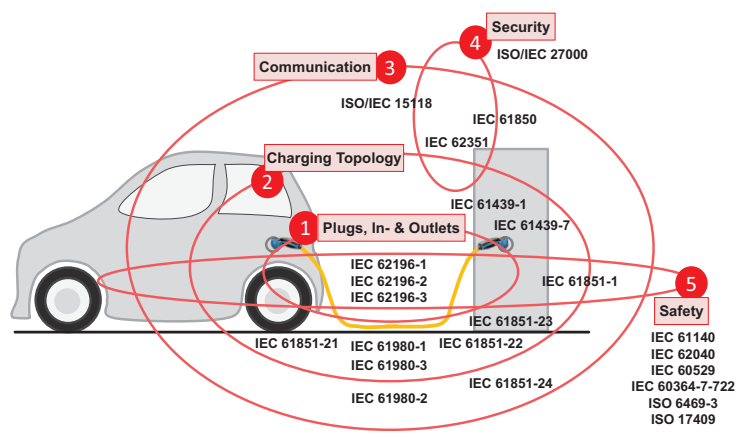

Figure 1: Digest of main ISO/IEC standards for the scope of the E-Mobility V2G Interface based on [3]

\subsection{Plugs (in- \& outlets)}

The IEC 62196 standards series is defined in TC23/SC23 and currently comprises three parts. The first part defines general requirements for dedicated plugs, socket outlets, vehicle connectors and vehicle inlets for interfacing a dedicated charging equipment with an EV. The current version was released end of 2011 and represents the second edition of this standard. However, work on the third edition has already started in the meantime. The second part defines the dimensional compatibility and interchangeability requirements for AC plugs (in- \& outlets). The standards defines three different types of connectors, type 1 for single phase charging supporting charging rates of up to $7.4 \mathrm{~kW}$. Whereas type 2 supports single and three phase charging, the latter with rates of up to $44 \mathrm{~kW}$. In January 2013 the European Commission declared the type 2 connector as a common basis for charging infrastructures and EVs in the European Union. Part three of the standard defines dimensional compatibility and interchangeability requirements for DC plugs (in- \& outlets) as well as Combo Plugs (combined AC \& DC plugs and in- \& outlets). Until today this part is still work in progress and not yet published as an international standard. The relevance of IEC 62196 regarding interoperability of the V2G interface is limited to physical specifications in terms of signaling pins and connector compatibility (physical layout \& dimensions).

\subsection{Charging Topologies}

Different charging topologies need to be considered for conductive AC- and DC-based dedicated charging equipments. Such EV charging equipments are defined in the IEC 61851 standards series. The first part describes general requirements for conductive charging systems. It applies to on-board and off-board AC and DC charging equipment and also to any additional services on the vehicle which may require electrical power when connected to the supply network. It defines four different charging modes starting from slow charging using household-type socket outlets to fast charging using an external charger. It also defines characteristics and operating conditions of the supply device and the connection to the vehicle as well as the operator's and third party's electrical safety. IEC 61851-1 specifically defines a safety-related low level signalling process based on a Pulse Width Modulation (PWM) signal indicating various EV connection states, supported charge currents and communication means. The PWM signal provides means for handling timecritical state changes, some of them even with respect to individual safety. Hence, IEC 61851-1 is a cross-cutting standard in terms of the previously mentioned domains, dealing with charging topologies, safety and communication (on a signalling level). The second edition was released end of 2010 but work is already ongoing for the third edition of this standard. The second part IEC 61851-21 describes technical require- 
ments relevant for EVs being connected to AC or DC EVSEs. Two further sub-parts of this standard detail the EMC requirements of on-board (IEC 61851-21-1) or off-board (IEC 61851-21-2) chargers. The following parts IEC 61851-22 and IEC 61851-23 detail specific requirements for AC- or DC-based EVSEs, respectively. Part 24 of IEC 61851 is described in section 2.3.

The charging topologies and relevant technical requirements for inductive charging equipments are defined in IEC 61980-1 where specific requirements on the magnetic field power transfer systems are detailed in IEC 61980-3.

General rules and definitions for service conditions and technical characteristics as well as construction and verification requirements for lowvoltage switch- and controlgear assemblies are defined in IEC 61439-1. However, this part of the standard cannot be used alone to specify an assembly or for the purpose of determining conformity because such compliance can only be achieved in combination with an additional relevant part of the IEC 61439 detailing a specific type of assembly; such being specified in Parts 2 onwards. For dedicated charging equipments IEC 61439-7 is foreseen but still work in progress.

\subsection{Communication}

In order to define the V2G Communication Interface the ISO/IEC 15118 Joint Working Group 1 (JWG1) was formed in 2009. Its purpose is to define a bidirectional IP-based communication protocol complementary to the low level signalling defined in IEC 61851-1. JWG1 started defining the first three parts in 2009. ISO/IEC 15118-1 defines all relevant terms for the V2G Interface and provides basic definitions and use cases being considered as part of the specification. It was released in early 2013 . The technical specifications of the standard for conductive charging are defined in ISO/IEC 15118-2 and ISO/IEC 151183 and are still work in progress. Part 2 defines the IP binding process, complete message flows and data types for AC and DC charging between

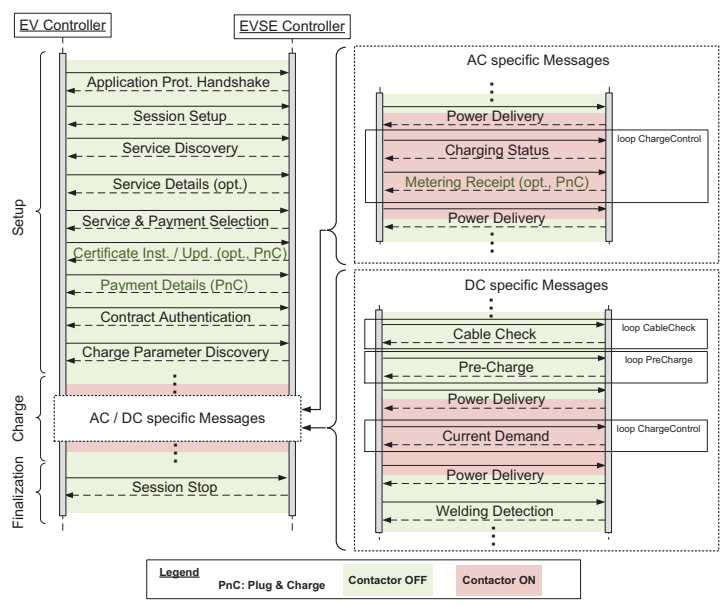

Figure 2: DIS ISO/IEC 15118-2 V2G Message Flows communication controllers in EVs (EVCC) and charge spots (SECC) as shown in Figure 2. Part 3 defines the physical and data link layer requirements of the powerline communication based carrier technology including the association process. The envisioned high level communication protocol will allow for advanced interaction between the grid and connected EVs for authentication \& authorization, accounting, load leveling management and further added-value services. The primary scope of the standard is limited to the communication between the EVCC and SECC as illustrated in Figure 3.

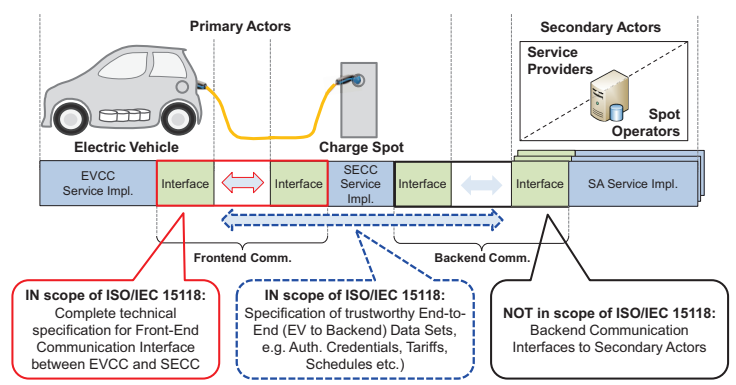

Figure 3: Scope of ISO/IEC 15118

Subsequently to the first three parts, the joint working group started new projects in 2012 to define conformance tests of the technical specifications as part of ISO/IEC 15118-4 (part 2 conformance tests) and ISO/IEC 15118-5 (part 3 conformance tests). Further work was also started for the definition of wireless physical and data link layers of the V2G Communication Interface in ISO/IEC 15118-8. Next to communication means for inductive charging scenarios, such an alternative carrier will also bring along new use cases even for conductive charging scenarios. A complementary set of use cases suitable for wireless carriers are therefore defined in ISO/IEC 15118-6. An additional alignment specification targeting document ISO/IEC 15118-7 will introduce all differences and new message sets compared to the core message specification (ISO/IEC 15118-2) in powerline communication based scenarios. However, all these parts are in very early stages of the standardization process. IEC 61851-24 defines the digital communication between a DC charging equipment and an EV for the control of the charging process. The standard is still work in progress and currently details various different technical approaches in its annex. One approach represents the CHAdeMO charging systems [4] which employs a CAN interface between the EV and the charging equipment. Another annex references the technical details defined in ISO/IEC 15118-2 and ISO/IEC 151183 without changing or extending the specification in any way. The same applies for the proposed work in IEC 61980-2 for inductive charging which is supposed to be referencing the upcoming parts 7 and 8 of the ISO/IEC 15118 series. However, at this time all standardization efforts regarding wireless communication for either inductive or conductive charging of EVs are in very early stages. 
Today, the IEC standards landscape does not cover a specific back-end protocol for EV charging infrastructures. One potential candidate may be IEC 61850 which focuses on grid automation for various types of grid assets. Its latest advancements in the direction of automation in the context of Distributed Energy Resources (DER) is particularly interesting for the E-Mobility domain. However, the scope of IEC 61850 is much broader and originates from the substation automation domain. The standards comprises more than ten parts where those of relevance for this work will be more closely discussed in section 4 .

\subsection{Security}

Due to the extended amount of Machine-toMachine (M2M) communication in the context of E-Mobility as introduced in 2.3, there is substantial need for information and communication security in the context of smart charging EVs. In contrast to conventional road vehicles, EVs regularly connect to public and heterogeneous infrastructures and exchange partly confidential information. For the OEM domain this is a fairly new use case scenario with special requirements in terms of information security as well as privacy. Hence, information security plays a key role in the definition of ISO/IEC 151182 . Next to securing the communication channel between the EVCC and the SECC through the establishment of a TLS channel, some endto-end security requirements are addressed between the EVCC and secondary actors, like charge spot operators, service providers, etc. (see Figure 3). Most dependencies on such external secondary actors are limited to authentication/authorization for service consumption, tariffing/billing issues or grid operation purposes like negotiation of dynamic grid limits. Message elements of ISO/IEC 15118-2 with relevance regarding these aspects are handled through endto-end security by XML security, particularly XML Signature [5] and XML Encryption [6] in the application layer.

IEC 61850 does not provide its own security mechanisms. It refers to IEC 62351 which consolidates security aspects for all communication standards within TC57. The security aspects being covered by IEC 62351 start at security policies and procedures like Role Based Access Control $(R B A C)$, but also include message and transport security as well as network- and media security. A very important aspect being required in IEC 61850 is RBAC on data objects and corresponding measures for intrusion detection. Such measures are defined as part of the Generic Security Application (GSAL) logical node whereas IEC 62351-8 defines corresponding roles of access and the underlying RBAC architecture. Similarly to ISO/IEC 15118, message and transport layer security include authentication of data access and transfer for routable network traffic based on SSL/TLS with digital signatures ensuring the prevention of eavesdropping, playback and spoofing.

ISO/IEC 27000 is typically referenced as common denominator for terminology in the scope of information security. The standard's series was started as a joint effort between ISO \& IEC defining the scope of Information Security Management Systems, not only for power systems.

\subsection{Safety}

In terms of safety requirements for $\mathrm{EV}$ charging infrastructures, IEC 61140 defines common aspects for the installation and equipment of electrical assemblies in order to ensure protection of persons and animals against electric shocks. It is intended to provide the fundamental principles and requirements which are common to electrical installations, systems and equipment or necessary for their co-ordination. Closely related to IEC 61140, IEC 60529 defines the degrees of protection which must be provided by enclosures of electric equipments. Extending the scope of these rather general safety standards, IEC 60364-7-722 particularly defines safety requirements for supply equipments of EVs in low voltage electrical installations. It furthermore also covers safety requirement for reverse energy flow from the EV back to public grid infrastructures. The work on this part of the standard is still in progress.

Related to maintenance work and also reverse energy flow, IEC 62040 is also in the scope of safety requirements for E-Mobility. It defines general and safety requirements for Uninterruptible Power Systems (UPS) with an electrical energy storage device in the DC link. The scope of the standard is applicable to movable UPS in low-voltage distribution systems which are furthermore intended to be installed in any operator accessible area or in restricted access locations. It specifies requirements to ensure safety for the operator, service personnel and any layman who may come into contact with the equipment.

On the EV side, ISO 6469-3 published in 2011 defines the safety specifications for EVs in order to ensure protection of persons inside and outside the vehicle against electric shocks. In this scope it details the specific requirements for electric propulsion systems and, if appropriate, conductively connected auxiliary electric systems. Furthermore, ISO 17409 defines safety requirements for the connection of an EV to an external electric power supply. This standard is still work in progress and only applies to on-board charging circuits. It is complementary to the definitions in the IEC 61851 series and focuses on the road vehicle's side.

\subsection{Back-End Communication Relevance}

The review of the E-Mobility standardization landscape from previous subsections allows us to assess the relevance of each domain regarding front- and back-end communication. Table 1 summarizes the result of this assessment according to the following scale: (- -) not relevant, (-) marginal relevance, $(\mathrm{o})$ neutral, $(+)$ relevant, $(++)$ substantial. 


\begin{tabular}{|l|l|l|}
\hline Domain & $\begin{array}{l}\text { Front-End } \\
\text { Comm. }\end{array}$ & $\begin{array}{l}\text { Back-End } \\
\text { Comm. }\end{array}$ \\
\hline Plugs (In- \& Outlets) & $(+)$ & $(-)$ \\
\hline Charging Topologies & $(++)$ & $(+)$ \\
\hline Communication & $(++)$ & $(++)$ \\
\hline Security & $(++)$ & $(++)$ \\
\hline Safety & $(+)$ & $(-)$ \\
\hline
\end{tabular}

Table 1: Relevance of E-Mobility Standardization Domains for V2G Front- \& Back-End Comm.

\section{Open Charge Point Protocol}

With the Open Charge Point Protocol (OCPP) a forum of European industry members was formed to define an open back-end protocol for charge spots which was initially started by e-laad in 2009 for their back-end system. The OCPP forum today has many members who agreed upon a common basis for their back-end protocol aiming at reducing overall integration and investment costs for charge spot development and operation. The initiative started with basic functionality like starting or stopping a charging transaction as well as basic asset management functionality for remote access $\&$ configuration means, diagnostics, firmware updates etc.

\subsection{Technical Principals of OCPP}

Version 1.5 of OCPP specifies operations between a charge spot and a central system, typically operated by one charge spot operator. Certain operations are defined to be invoked on the charge spot whereas others are considered for invocation at the central system only. In order to allow such bidirectional service invocation, two services are defined: Charge Point Service (CPS) and Central System Service (CSS). Figure 4 illustrates the overall concept of OCPP 1.5.

The OCPP specification only allows RequestResponse Message Exchange Patterns (MEP) between both services. Other MEPs like OneWay, Notification or Solicit-Response are not defined. Both OCPP services are based on Web Services paradigms adopting the SOAP 1.2 message framework. The interface description for these services is based on a WSDL which is published on the OCPP website. According to the specification the communication channel should be secured by HTTPS over SSL/TLS. In Figure 5 the complete OCPP 1.5 protocol stack is shown.

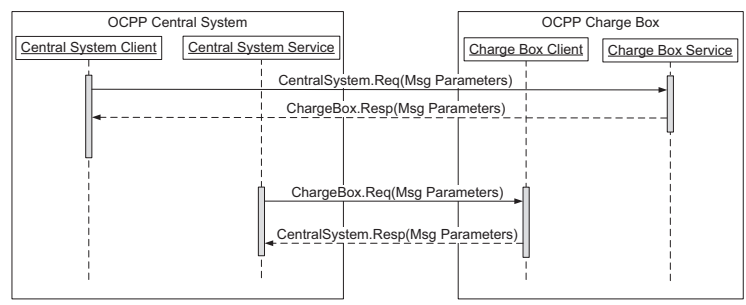

Figure 4: OCPP 1.5 Architecture and MEPs

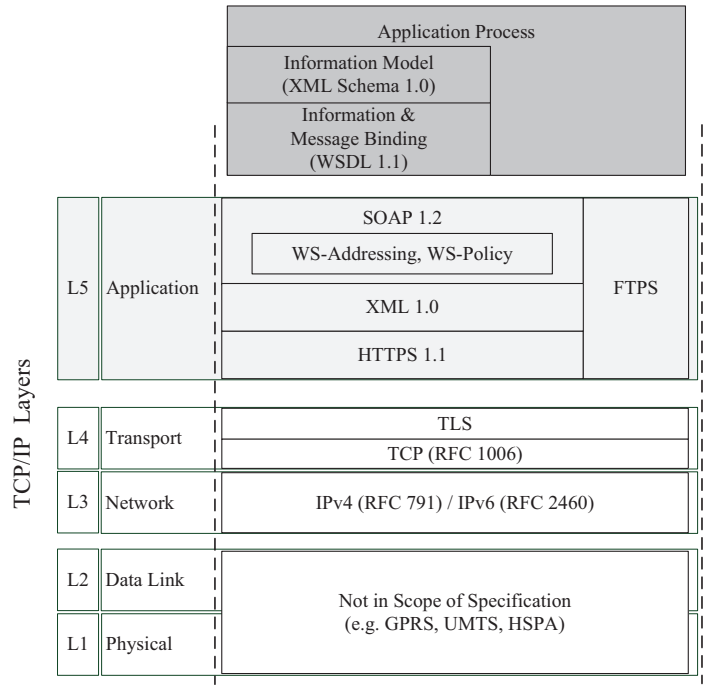

Figure 5: OCPP 1.5 Protocol Stack

The OCPP specification currently defines the following list of largely self explanatory message sets which are shown in Table 2.

All these messages are defined in the WSDL description and describe atomic operations between the invoking client and its corresponding service. Other than WS-based message transactions, OCPP also defines FTP file transfers (see Figure 5) as part of the firmware update process (FTP download triggered by Update Firmware) and for diagnostic purposes (FTP upload triggered by Get Diagnostics).

\begin{tabular}{|l|l|}
\hline Service & Supported Operations \\
\hline OCCP Central & Authorize \\
System Service & Boot Notification \\
(CSS) & Data Transfer (Vendor-specific) \\
& Diagnostics Status Notification \\
& Firmware Status Notification \\
& Heartbeat \\
& Meter Values \\
& Start / Stop Transaction \\
& Status Notification \\
\hline OCCP Charge & Cancel Reservation \\
Point Service & Change Availability \\
(CPS) & Change Configuration \\
& Clear Cache \\
& Data Transfer \\
& Get Configuration \\
& Get Diagnostics \\
& Get Local List Version \\
& Remote Start / Stop Transaction \\
& Reserve Now \\
& Reset \\
& Send Local List \\
& Unlock Connector \\
& Update Firmware \\
\hline
\end{tabular}

Table 2: Supported Operations in OCPP 1.5 

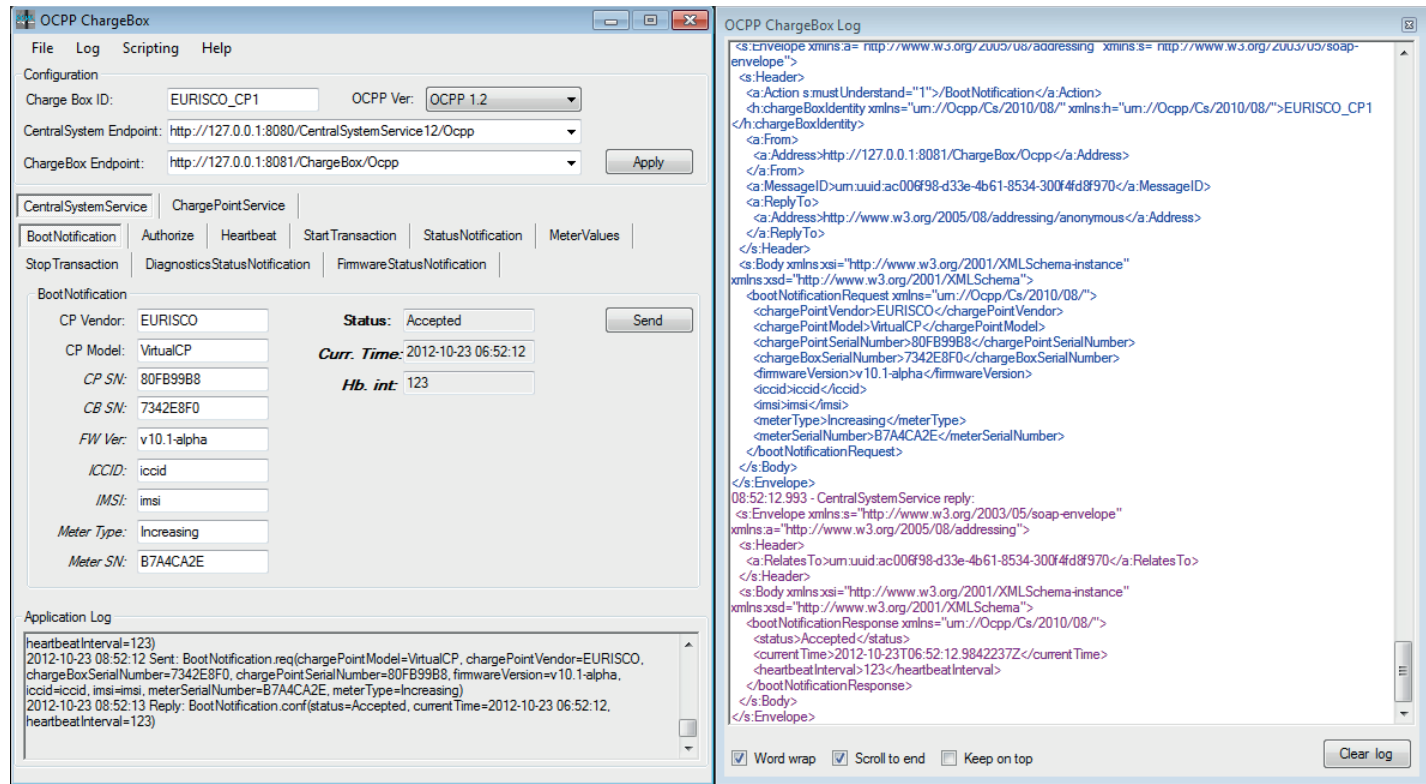

Figure 6: Screenshot of EURISCO OCPP Test Bench Software with SOAP Messaging Log

\subsection{Evaluation of OCPP 1.5}

Based upon the experience the authors gathered during the implementation process of OCPP 1.5 clients and services we provide our lessons learned from following three perspectives: Functional scope/limitations of OCPP, issues and drawbacks from an operational / technical point of view, and maturity of the OCPP 1.5 specification.

\subsubsection{Functional Scope and Limitations}

Starting with the functional scope of OCPP 1.5 we found in section 3.1 that in its current form OCPP is highly tailored towards the charge spot operator's business. It fulfills all main requirements regarding the charge process, such as authentication and authorization, transaction handling, metering, reservation of a charge spot. It also supports maintenance tasks like status notifications, configuration and firmware handling, diagnostics, and internal support functions like resets, clearing of caches etc. It provides means for vendor-specific and therefore proprietary extensions for an operator's internal use. However, grid-related services, e.g. demand management by utilizing the functionality of ISO/IEC 15118 are currently missing. Smart charging of EVs in the sense of a grid service is therefore not yet considered in OCPP 1.5. The specification furthermore does not consider Role Based Access Control (RBAC) which might become essential in the future.

\subsubsection{Technical Issues and Drawbacks}

The use of SOAP-over-HTTP has many advantages in terms of the software development process for charge points due rich tool support, as well as out of the box syntactic interface conformance based upon the provided WSDL definition. However, the use of SOAP-over-HTTP also leads to huge message overheads which may be an issue for some operators and could be optimized without loosing out on convenience of Web Services. Particularly in case of wireless back-end communication links like cellular radio access and in case of large scale charging infrastructures, this issue might lead to rather expensive maintenance costs for operators. By utilizing presentation layer formats like Efficient XML Interchange (EXI) these issues could be alleviated. As the authors already showed in [8] using EXI provides a compression rate of up to $5-10 \%$ for the message payload size of V2G messages in ISO/IEC 15118. Similar improvement would be feasible in case of using EXI for OCPP. Further improvement could be yield by opting for ICMP ping-like heartbeat messages instead of using a SOAP-based Request-Response MEP.

\subsubsection{Specification Maturity}

Based upon our own experience with implementing OCPP we rate the maturity of the specification as average. Requirements should be clearly identified by a numbering scheme in order to allow for requirement traceability in the development as well as in the testing phase. Conformance and interoperability issues are quite common for various OCPP implementations since the specification is quite vague e.g. in terms of for handling security. With the help of example stubs and skeletons provided by the OCPP forum as well as third party conformance testing tools like EURISCO's OCPP TestBench (see Figure 6), concrete conformance and testing tools are available today, alleviating the lack of a concrete conformance test specification. 


\section{IEC 61850 - Communication Networks and Systems for Power Utility Automation}

IEC 61850 represents a core standard for future Smart Grid deployments and plays a particularly important role in the grid automation domain according to the IEC roadmap [2]. Originating from the domain of substations, the scope of IEC 61850 was continuously extended integrating various types of Intelligent Electronic Devices (IEDs) in the energy distribution process, especially Distributed Energy Resources (DERs), like photovoltaic or combined heat \& power, etc. IEC 61850 defines a set of abstract objects and services virtualizing the state as well as functionality of an IED in an abstract model making them accessible to authorized parties.

\subsection{Technical Principles of IEC 61850}

The modeling of an IED in IEC 61850 is strictly object-oriented and driven by its components and their corresponding functionality. Every abstract model of an IED is therefore composed of a set of Logical Devices and Logical Nodes. Logical Devices represent components of IEDs whereas the functionality of each Logical Device is described by a set of Logical Nodes. Each Logical Node refers to an Object Information Model (OIM) which defines a concrete set data objects and data attributes for the node. In order to interact with an IED, each Logical Node exchanges information through an Abstract Communication Service Interface (ACSI) being assigned to certain roles for Role Based Access Control (RBAC) on data objects and furthermore characterized by Quality of Service criteria. An overview on the abstract model of an IED is shown in Figure 7. IEC 61850 builds upon typical communication network protocol stacks like TCP/IP and/or Ethernet but may also be implemented over differ-

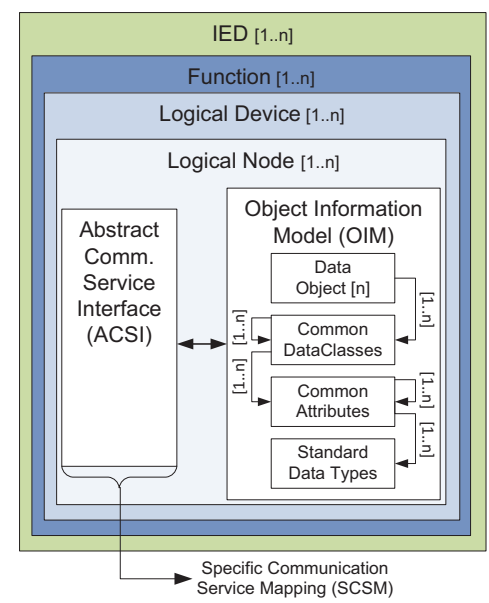

Figure 7: Virtualization of Intelligent Electronic Devices in IEC 61850

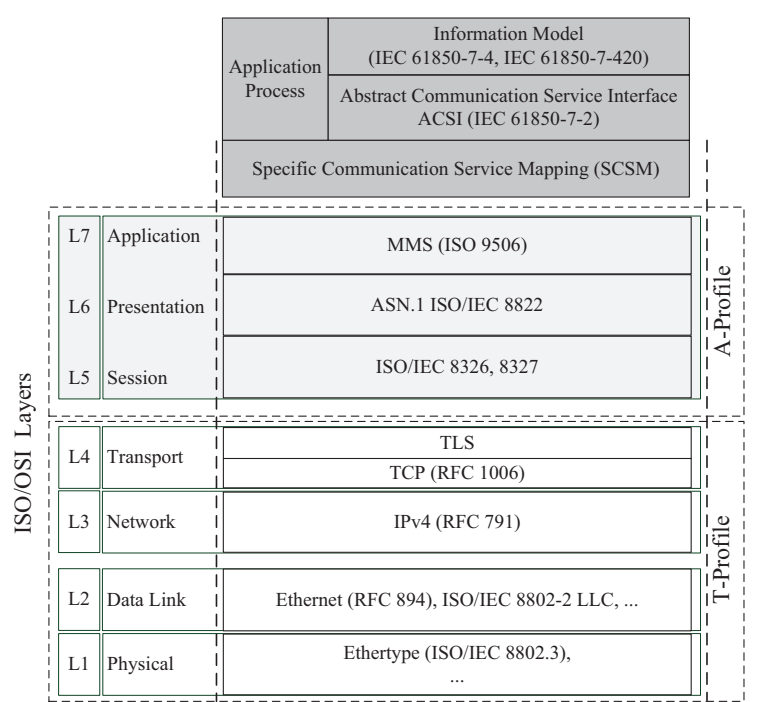

Figure 8: IEC 61850 Protocol Stack for MMS-based SCSM

ent protocol stacks. It supports both client-server and peer-to-peer communication paradigms. The client-server approach is typically used for less time critical communication services utilizing TCP/IP, whereas the peer-to-peer approach is typically used for real-time communication services directly using Ethernet, e.g. GOOSE. In order to define the concrete interface to networking protocols and frame formats, the ACSI is extended by Specific Communication Service Mappings (SCSM). One SCSM example for the client-server based approach is the Manufacturing Message Specification (MMS) which builds upon a TCP/IP stack and is defined in IEC 618508-1 [9]. The corresponding protocol stacks for this specific SCSM are illustrated in Figure 8.

As illustrated in Figure 8 the ACSI definition allows for a high level of abstraction between the definition of logical nodes including their communication services and concrete implementations for underlying networking protocol stacks. It is therefore essential in terms of extendability towards other potential protocol bindings, like e.g. Web Service-based SCSMs which are currently under development for IEC 61850-8-2, see also IEC 61400-25-4 [10].

\subsection{State of E-Mobility in IEC 61850}

In today's published series of IEC 61850 standards, EVs are not yet considered in IEC 618507-420 [11] as Distributed Energy Resources (DERs), even though EVs could act as energy storage for volatile energy generators such as wind power plants or PV plants. The authors already proposed an object model for electric mobility in [12] which was furthermore published in more detail as IEC Technical Report 61850-90-8 in the meantime [13]. As shown in these previous works the proposed object model satisfies the requirements of ISO/IEC 15118 as well as IEC 61851-1 in terms of monitoring and 
parametrization of an EV charging process. For a charge spot operator such functionality may become crucial in the future in order to provide grid services to other stakeholders e.g. in the form of Demand Response Mgmt. (DRM) or as part of a technical head of a Virtual Power Plant (VPP). This section briefly summarizes the underlying approach.

The first step in the modeling process was the analysis of information being provisioned according to the E-Mobility standardization landscape described in detail in section 2. As part of these standards a multitude of control and monitoring information is exchanged between technical components being involved in the charging process in order to ensure an automated and safe charging process. However, for managing EVs as DERs in the grid only a subset of this information is necessary and must be identified. In order to ensure a scalable system design it is explicitly required to reduce level of detail in terms of information provisioning for upstream stakeholders, e.g. infrastructure operators.

The second step in the modeling process addressed the operators view on an interconnected EV. In accordance with the first step, necessary input and output parameters for monitoring and control purposes must be identified and modeled accordingly. The modeling approach taken for this work adheres to IEC 61850-7-420 guidelines. Figure 9 provides an overview which entities are involved in the information provisioning process, what information originates from which entity and how this information is finally mapped to the proposed E-Mobility Object Model.

The diamond shaped elements in the diagram differentiate three different types of information: Config, Setting, and Measurement. Configs (blue) describe persistent information, e.g. defining hardware installation characteristics. Settings

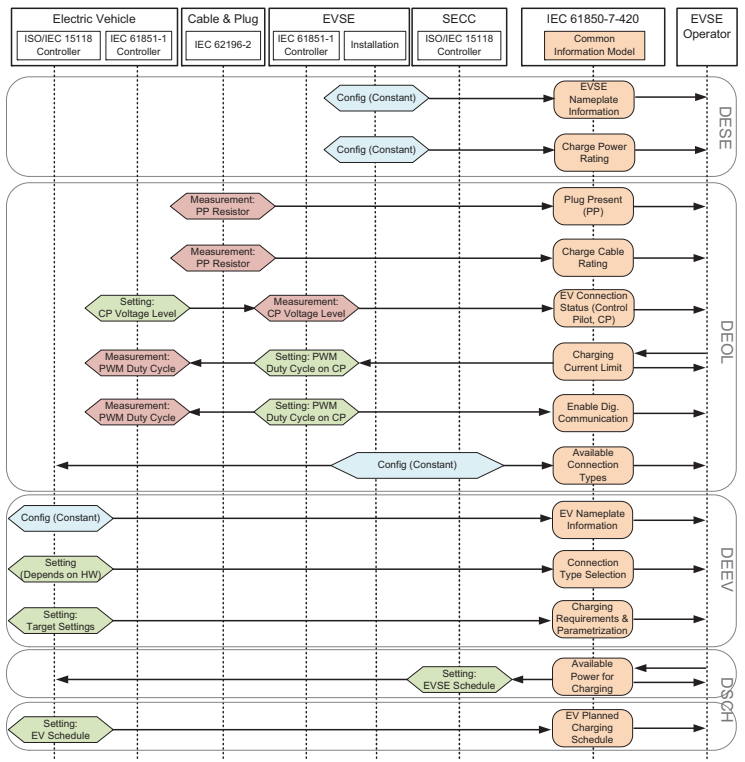

Figure 9: E-Mobility Object Modeling Approach in IEC TR 61850-90-8 [12] (green) describe dynamic configuration information of an entity which may change over time and may be influenced from a secondary actor, like the EVSE operator. Measurements (red) are observed at the respective entity and also represent dynamic information. The actual information being provided by the respective entities is summarized in the common information model (orange) in Figure 9 and is mapped to the following three newly defined LNs of the E-Mobility Object Model:

1. DESE: This LN represents an EVSE which may house several outlets and contains information related to monitoring and controlling of the EVSE.

2. DEOL: This $\mathrm{LN}$ represents an individual EVSE outlet and contains information related to monitoring and controlling of the outlet.

3. DEEV: This LN represents a connected EV and contains information on an EV connected to an EVSE. If the connection / plug status indicates that no EV is connected the DEEV LN is unregistered.

The DSCH LNs in Figure 9 are re-used from IEC 61850-7-420 and cover the two way charge schedule negotiation handshake of ISO/IEC 15118 (see also [14]). In addition and as shown in Figure 10, a charging infrastructure operator may include further LNs known from the already existing portfolio of LNs in IEC 61850$7-2,-7-4,-7-420$ if necessary in order to represent the charging infrastructure's setup.

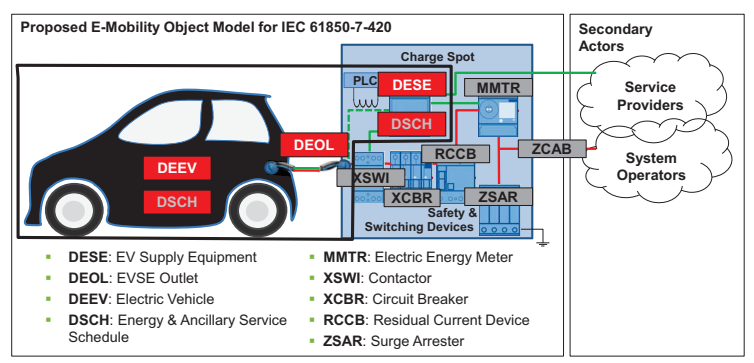

Figure 10: Example AC-Charging Deployment Scenario of the E-Mobility Object Model

This abstract model of the charging infrastructure allows for flexible physical designs of EV-based DERs. The selection of LNs on the one hand and adhering to their specification on the other ensures harmonized information models between involved actors, on top of independent underlying protocol bindings corresponding to the ACSI and SCSM.

Demand \& supply management is provided by setting the charge spot's DSCH Logical Node for the Local Load Limit Profile as well as observing the EV's virtual DSCH Logical Node for the Local Load Reservation Profile. Depending on whether the EVSE controller acts upon one individual outlet or implements a load management 
for several outlets, the DSCH schedules either refer to a single outlet and its corresponding EV or an aggregate of all managed outlets and the corresponding set of EVs. The integrated sequence including the ISO/IEC 15118 charge parameter negotiation process as well as handling of the DSCH Local Load Limit Profile and the DSCH Local Load Reservation Profile is detailed in Figure 11 .

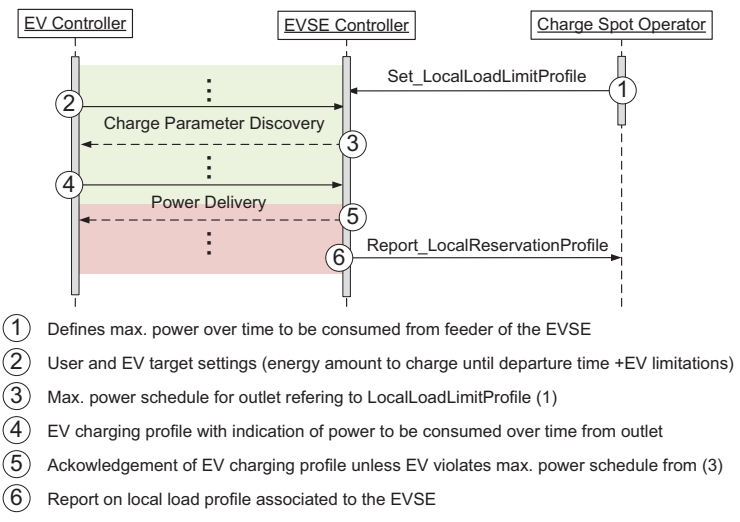

Figure 11: Load Management for Smart Charging with ISO/IEC15118 and E-Mobility Object Model according to IEC TR 61850-90-8

Based upon these principles, the current version of the proposed E-Mobility Object Model in IEC 61850-80-9 represents the functions summarized in Table 3:

\begin{tabular}{|l|l|}
\hline Service & Supported Operations \\
\hline IEC 61850 & Get EVSE (\& EV) Nameplate \\
E-Mobility & Get Charge Power Rating \\
Charge Point & Get Charge Cable Rating \\
Service & Get Plug Present \\
& Get (Available) Connection Type \\
& Get State (A,B,C,D,E,F) \\
& Enable / Disable Digital Comm. \\
& Get User Target Settings (Energy \\
& Amount, Departure Time) \\
& Get EV Charge Power Rating \\
& Set Local Load Limit Profile \\
& (EVSE/Station limits) \\
& Report Local Reservation Profile \\
& (EV/EV Pool Reservation) \\
\hline
\end{tabular}

Table 3: Overview of Supported Operations of IEC 61850-90-8 E-Mobility Object Model

Note, Table 3 only refers to newly defined LNs as part of [13] that are also shown in Figure 9. Other services and respective functions may be added by the means of already existing LNs according to IEC 61850-7-2, -7-4, -7420 , e.g. metering capability through the Logical Node MMTR.

\subsection{Evaluation of IEC 61850 for E-Mobility}

In line with the evaluation of OCPP in section 3.2, we look again at the following list of criteria: Functional scope/limitations of IEC 61850 in E-Mobility context, problems and drawbacks from an operational / technical point of view, and maturity of the IEC 61850 \& E-Mobility Object Model specification.

\subsubsection{Functional Scope and Limitations}

In contrast to OCPP, the functional scope of the IEC 61850 E-Mobility Object Model is tailored towards grid services but at the same time lacks many other operational aspects of the charge spot operator's business. The IEC 61850-90-8 design approach considered from the very beginning all other ISO/IEC V2G standards and ongoing standardization efforts. Hence, it fulfills all smart charging requirements which are also considered in the current proposition of e.g. ISO/IEC 151182. It is also extensible by other existing LNs from the Part 7 series of IEC 61850. However, basic functionality for charge spot operators like authenticating an user who wants to charge at a charge spot is missing because it was considered out of scope of IEC 61850 from the very beginning and delegated to other specifications, e.g. IEC 61970, OCPP etc. In general, IEC 61850 provides in conjunction with IEC 62351 Role Based Access Control (RBAC) to operational grid stakeholders which might furthermore become essential in multi-use scenarios of charging infrastructures.

\subsubsection{Technical Issues and Drawbacks}

The issue with IEC 61850 today is that coming from the substation domain it is not well integrated in heterogeneous environments due to its currently very restrictive and inflexible binding technology which is based on the Manufacturing Message Specification (MMS) dating back to the 1990s. New binding mechanisms based on Web Service technologies like SOAP or REST would allow much better integration with assets from other domains as well as common IT back-end systems and would furthermore simplify development for spot operators due to a much larger tool-chain support. These aspects including a set of solution concepts are also already detailed by the authors in [15] and supported by others in [16] and [17]. Working Group 17 of IEC TC57 is also already investigating WS-based bindings for IEC 61850 and successfully proposed a respective new work item proposal for IEC 618508-2 in 2012. When moving towards Web Service based bindings, the use of a suitable presentation layer technologies should also be considered as previously also indicated as potential improvement for OCPP in section 3.2. 


\subsubsection{Specification and Standard Maturity}

The proposed E-Mobility Object Model for IEC 61850 was published as technical report in TC57. It is currently revised to align with ongoing standardization processes of surrounding standards in the E-Mobility landscape, e.g. ISO/IEC 15118. In general, IEC 61850 has a very long history and builds upon a mature set of specifications. However, the complexity of the standard, its long learning curves, and the complex standardization processes certainly are disadvantages. New Web Servicebased bindings might help to simplify application of IEC 61850 and gain larger audiences in the industry. Especially in the domain of DERs it may help for quicker adoption rates, like it has already been adopted for wind power generation in IEC 61400-25-4.

\section{Lessons Learned}

The previous analysis showed, that the technical approaches taken in OCPP 1.5 and IEC 61850 with the proposed E-Mobility Object Model are quite different. Also from a functional point of view there are only marginal overlaps between both candidates. OCPP 1.5 clearly addresses the business domain of charge spot operators which are not necessarily directly interested in smart charging of EVs. IEC 61850 on the other side is all about grid automation and therefore closer to grid stakeholders like plant and distribution system operators. With the trend towards an increasing amount of distributed energy supply, the need for integrating EVs as DERs with a certain storage capacity and controllable demand curves will become more crucial in the long-term.

From a technical perspective our evaluation on OCPP and IEC 61850 for smart charging of EVs results in the following three feasible scenarios:

- Leveraging the IEC 61850 E-Mobility Object Model as Meta-Model for enabling Smart Charging in OCPP.

- Integrated approach for OCPP and IEC 61850 based on new IEC 61850-8-2 Web Service Binding.

- Outlook on future releases.

\subsection{IEC 61850 E-Mobility Meta-Model}

The first option describes the situation where one or several implementations exist using the IEC 61850 E-Mobility Object Model as MetaModel and abstract service interface but without the Specific Communication Service Mapping (SCSM) according to IEC 61850. This approach would ensure the use of a harmonized and commonly agreed information model across various back-end communication protocols. However interoperability would fail on the interface level due to the lack of vertical integration of different SCSMs.

\subsection{OCPP and IEC 61850 Integration}

As described previously, the need for a Web Service binding for IEC 61850 becomes more and more important with new sorts of DER types being added to the IEC 61850 portfolio. Since OCPP is already based on a SOAP-based message framework and binding mechanism, it would be attractive for charge spot operators to use the same message framework and communication stack and integrate both OCPP and IEC 61850. The advantage of such a solution would be, that EV charging infrastructures could still be integrated as portfolio enhancement into other IEC 61850-based pools of DERs, like combined heat \& power, PV etc. without breaking either the IEC 61850's or OCCP's paradigms.

In order to combine the existing functionality of OCPP with automated grid services through smart charging of EVs according to [13], additional service(s) based on the IEC 61850 E-Mobility Object Model must be deployed on the charge spot, next to the OCPP Charge Point Service. Figure 12 illustrates the overall approach. The WSDL of the new IEC 61850 services corresponds to the IEC 61850 ACSI and maps the E-Mobility Object Model which is defined in XML Schema to the supported set of operations and messages (WS-based SCSM). The applicable set of operations resulting from a combined setup with OCPP 1.5 and the IEC 61850 E-Mobility Object Model would be a joint version of Tables 2 and 3 .

\subsection{Outlook}

The market may also develop in an entirely different direction based upon a different/new approach not considered in this work. At the time of this writing OCPP 2.0 is also being prepared for release. According to the OCPP roadmap, many of the issues highlighted in this paper are being addressed as part of the new release. Most importantly, OCPP 2.0 will integrate support for ISO/IEC 15118, which marks the most important point of criticism in this work. However, since OCPP is solely tailored towards the management and operation of EV charging infrastructures, it will not provide seamless integration with other types of DERs in the future as opposed to IEC 61850 . The same remains true for all other types of dedicated charging infrastructure back-end protocols. A plant operator who is envisioning to consolidate demand and supply from various heterogeneous sources, e.g. VPP, would need to support a multitude of protocol stacks coming along with an additional processing burden for the plant controller as well as potential interoperability issues and versioning conflicts. This sought of software fragmentation would furthermore complicate the software development and maintenance processes for the controller. In such scenarios, the option in 5.2 with support of IEC 61850 would be beneficial, since all tasks could be handled according to the same paradigms set by IEC 61850 across all types of DERs being used throughout a heterogeneous plant setup. Hence, operators who in the 


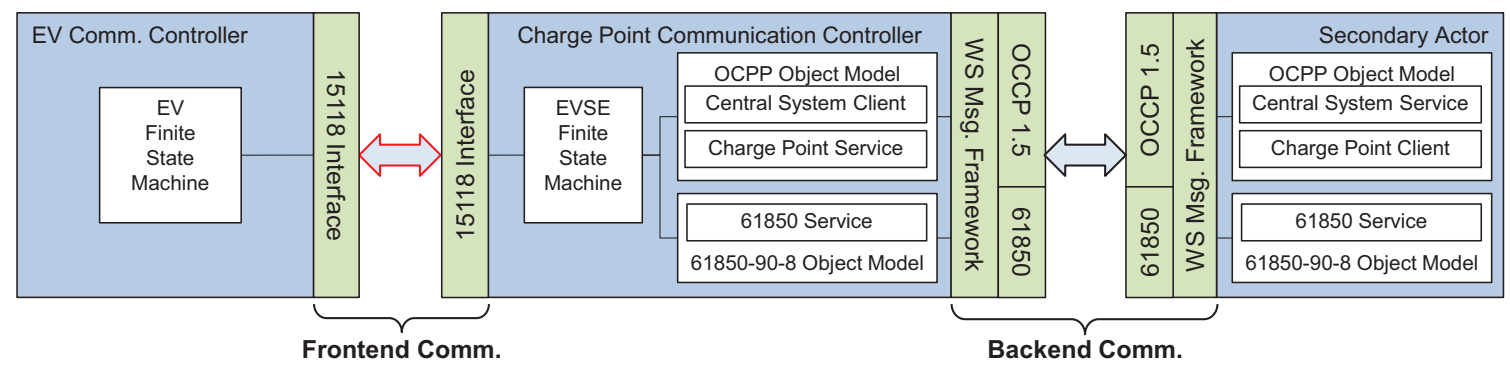

Figure 12: Combined OCPP and IEC 61850 Smart Charging Setup for ISO/IEC 15118

long run want to support advanced and in particular heterogeneous DER setups are well advised to consider such aspects from the very beginning.

\section{Acknowledgments}

The work in this paper was partly funded by the European Commission as part of the 7th Framework Programme with grant agreement number 285586. The authors would like to thank our partners in the corresponding EU research project e-DASH (www.edash.eu).

\section{References}

[1] European Parliament and the Council of the European Union, Directive 2009/28/EC on the Promotion of the use of energy from renewable sources and amending and subsequently repealing Directives 2001/77/EC and 2003/30/EC, 23 April 2009.

[2] SMB Smart Grid Strategic Group (SG3), IEC Smart Grid Standardization Roadmap - Edition 1.0 , June 2010.

[3] Nationale Plattorm Elektromobilitt (NPE), Die deutsche Normungs-Roadmap Version 2 (German Standardization Roadmap V2), [Online] available at: http://www.elektromobilitaet.din.de, accessed on 14.02.2013

[4] CHAdeMO Association, CHAdeMO Specification Version 1.0.0, Published 31.01.2012.

[5] Eastlake, Donald et al., XML Signature Syntax and Processing Version 1.1, W3C Recommendation 11 April 2013.

[6] Eastlake, Donald et al., XML Encryption Syntax and Processing, W3C Recommendation 10 December 2002.

[7] OCPP Forum, Open Charge Point Protocol, [Online] available at: http://www.ocpp.nl, accessed on 14.02.2013.
[8] Schmutzler, Jens; Wietfeld, Christian Analysis of Message Sequences and Encoding Effciency for Electric Vehicle to Grid Interconnections, Proceedings of IEEE Vehicular Networking Conference (VNC) 2010, Jersey City, New Jersey, USA, 2010.

[9] IEC TC57 IEC IS 61850-8-1 Communication networks and systems in substations - Part 81: Specific Communication Service Mapping (SCSM) - Mappings to MMS (ISO 9506-1 and ISO 9506-2) and to ISO/IEC 8802-3, International Electrotechnical Commission (IEC), Geneva, Switzerland, September 2011.

[10] IEC TC/SC 88 Wind turbines Part 25-4: Communications for monitoring and control of wind power plants Mapping to communication profile, Geneva, Switzerland, October, 2008.

[11] IEC TC57 IEC IS 61850 Communication networks and systems for power utility automation - Part 7-420: Basic communication structure - Distributed energy resources logical nodes, International Electrotechnical Commission (IEC), Geneva, Switzerland, June, 2009.

[12] Schmutzler, Jens; Andersen, Claus Amtrup; Wietfeld, Christian Distributed Energy Resource Management for Electric Vehicles using IEC 61850 and ISO/IEC 15118, Proceedings of 8th IEEE Vehicle Power and Propulsion Conference (VPPC), pp.1457-1462, Seoul, South Korea, 9-12 Oct. 2012.

[13] IEC TC57 WG17 IEC TR 61850-90-8 Communications Systems for Distributed Energy Resources Part 90-8: Object Model for Electric Mobility, May, 2012.

[14] Schmutzler, Jens; Wietfeld, Christian; Jundel, Sven; Voit, Stephan A mutual Charge Schedule Information Model for the Vehicle-to-Grid Communication Interface, Proceedings of 7th IEEE Vehicle Power and Propulsion Conference (VPPC), pp.1-6, Chicago, IL, USA, Sept. 2011. 
[15] Schmutzler, J.; Groning, S.; Wietfeld, C. Management of Distributed Energy Resources in IEC 61850 using Web Services on Devices, 2011 IEEE International Conference on Smart Grid Communications (SmartGridComm), pp.315-320, 17-20 Oct. 2011.

[16] Pedersen, A.B.; Hauksson, E.B.; Andersen, P.B.; Poulsen, B.; Trholt, C.; Gantenbein, D. Facilitating a Generic Communication Interface to Distributed Energy Resources: Mapping IEC 61850 to RESTful Services, 2010 First IEEE International Conference on Smart Grid Communications (SmartGridComm), pp.61-66, 4-6 Oct. 2010.

[17] Sucic, S.; Bony, B.; Guise, L. Standardscompliant event-driven SOA for semanticenabled Smart Grid Automation: Evaluating IEC 61850 and DPWS Integration, 2012 IEEE International Conference on Industrial Technology (ICIT), pp.403-408, 19-21 March 2012.

\section{Authors}

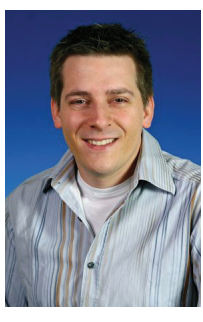

Jens Schmutzler is a senior researcher at the Communication Networks Institute (CNI) at the TU Dortmund University, Germany. He is actively involved in national and international research projects on interoperability of EVs and charging infrastructures and coordinates the ICT for Energy Systems research group at the CNI (currently 10 staff members). He is an active member of DIN (German Standardization Body) and a delegate in the Joint Working Group for standardization of the Vehicle-to-Grid Communication Interface (ISO/IEC 15118). He also contributed to WG17 of TC57 in IEC 61850 targeting an object model for e-mobility for ICT integration of EVs into the Smart Grid. His research background is embedded Web Services and their impact on constrained communication networks. Jens Schmutzler is a Dipl.-Ing. of TU Dortmund University and studied one year at the National University of Singapore (NUS).

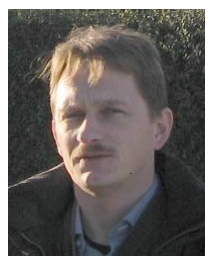

Claus Amtrup Andersen graduated from OTS, Denmark in 1993 with a degree in embedded hardware and software design. He has worked as Manager for EURISCO since 1994. Claus Amtrup Andersen is active member of IEC TC57 WG17 and IEC TC69 WG4 incl. JWG ISO/IEC 15118. Claus Amtrup Andersen has been PT-leader of the M/468 Focus group for E-mobility data communications and is now WG-leader of the CENELEC WG Smart Charging under EM-CG and SG-CG. As reporter between M/468 and M/490, Claus Amtrup Andersen is also member of the Steering group for the Smart Grid Coordination Group under CEN/CENELEC.

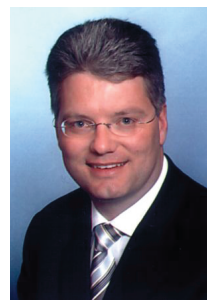

Christian Wietfeld received his Dipl.-Ing. and Dr.-Ing. degrees from RWTH Aachen University. $\mathrm{He}$ is a full professor and head of the Communication Networks Institute (CNI) of TU Dortmund University, Germany. For 20 years he has initiated and contributed to national and international research and development projects on wireless data communication systems in academia and industry. He has published over 165 peer-reviewed publications and holds several patents. For his contributions to the standardization of next generation mobile network architectures, he received in 1999 an ITU-T outstanding contribution award. His current research interests include the system design, modeling and performance evaluation of communication networks in challenging environments. Christian Wietfeld is a Senior Member of IEEE and chairs the IEEEs German sister organization VDE/ITG committee on Communication Networks and Systems. 